\title{
Photothermal killing of Staphylococcus aureus using antibody-targeted gold nanoparticles
}

\author{
This article was published in the following Dove Press journal: \\ International Journal of Nanomedicine \\ 18 March 2015 \\ Number of times this article has been viewed
}

\author{
Nancy J Millenbaugh' \\ Jonathan B Baskin' \\ Mauris N DeSilva' \\ W Rowe Elliott ${ }^{\prime}$ \\ Randolph D Glickman² \\ 'Maxillofacial Injury and Disease \\ Department, Naval Medical Research \\ Unit San Antonio, Joint Base San \\ Antonio-Fort Sam Houston, TX, USA; \\ ${ }^{2}$ Department of Ophthalmology, The \\ University of Texas Health Science \\ Center at San Antonio, San Antonio, \\ TX, USA
}

Purpose: The continued emergence of multidrug resistant bacterial infections and the decline in discovery of new antibiotics are major challenges for health care throughout the world. This situation has heightened the need for novel antimicrobial therapies as alternatives to traditional antibiotics. The combination of metallic nanoparticles and laser exposure has been proposed as a strategy to induce physical damage to bacteria, regardless of antibiotic sensitivity. The purpose of this study was to test the antibacterial effect of antibody-targeted gold nanoparticles combined with pulsed laser irradiation.

Methods: Gold nanoparticles conjugated to antibodies specific to Staphylococcus aureus peptidoglycan were incubated with suspensions of methicillin-resistant and methicillin-sensitive S. aureus (MRSA and MSSA). Bacterial suspensions were then exposed to 8 ns pulsed laser irradiation at a wavelength of $532 \mathrm{~nm}$ and fluences ranging from 1 to $5 \mathrm{~J} / \mathrm{cm}^{2}$. Viability of the bacteria following laser exposure was determined using colony forming unit assays. Scanning electron microscopy was used to confirm the binding of nanoparticles to bacteria and the presence of cellular damage.

Results: The laser-activated nanoparticle treatment reduced the surviving population to $31 \%$ of control in the MSSA population, while the survival in the MRSA population was reduced to $58 \%$ of control. Significant decreases in bacterial viability occurred when the laser fluence exceeded $1 \mathrm{~J} / \mathrm{cm}^{2}$, and this effect was linear from 0 to $5 \mathrm{~J} / \mathrm{cm}^{2}\left(r^{2}=0.97\right)$. Significantly less bactericidal effect was observed for nonfunctionalized nanoparticles or functionalized nanoparticles without laser activation.

Conclusion: Laser-activated nanoparticles targeted to $S$. aureus surface antigens significantly reduced the percentage of viable organisms and represents a promising new treatment modality that could be used either alone or as an adjunct to existing, conventional antibiotic therapy.

Keywords: MRSA, bacteria, pulsed laser, nanospheres, photoacoustic

\section{Introduction}

The development of multidrug resistance in pathogenic bacteria is a significant public health risk across the world. Methicillin-resistant Staphylococcus aureus (MRSA) has become one of the pathogens of greatest concern due to its ability to cause a wide range of infections ranging from localized skin conditions to life threatening pneumonia and sepsis and its high prevalence in hospital- and community-associated settings. ${ }^{1}$ Skin and soft tissue infections (SSTIs) are the most common manifestation of MRSA infection in the community setting. ${ }^{2}$ Recent studies found that MRSA now accounts for $59 \%$ of SSTIs presenting to emergency departments in the US, ${ }^{3}$ and the national cost associated with community-acquired MRSA SSTIs ranges from \$108 to \$343 million annually. ${ }^{4}$ The emergence of community-acquired MRSA infections over the last decade has been notable because those affected are typically young, healthy individuals without any apparent risk factors, ${ }^{2}$ the infecting strains tend to be more
Correspondence: Nancy J Millenbaugh 3650 Chambers Pass, Building 36I0, Joint Base San Antonio-Fort Sam Houston,

Texas 78234-63I5, USA

Tel +l 2105397133

Fax + | 21053993||

Email nancy.j.millenbaugh.civ@mail.mil 
virulent than nosocomial isolates, ${ }^{5}$ and it correlates with a coincident increase in the total number of hospitalizations in the affected patients. ${ }^{6}$ Current trends indicate the expanding reservoir of MRSA in the community is likely to become a source for recurrent transmission into hospitals where it would put many more patients at risk for developing highly virulent and multidrug resistant infections. ${ }^{7}$ Due to the steady decrease in the rate of new antibiotics reaching the market, an urgent need exists for the development of alternate therapeutic approaches.

One potential strategy for circumventing multidrug resistance mechanisms that has gained interest in recent years is the use of light-based therapies to induce chemical or physical damage to the bacteria. ${ }^{8-13}$ While photodynamic therapy involving use of photosensitizing dyes has been widely shown to be effective at killing bacteria via generation of reactive oxygen species, ${ }^{13}$ use of metallic nanomaterials as photoabsorbers offers many advantages over this approach. Gold nanoparticles (GNPs) in particular are viewed as a promising platform for light-based therapies because they are predicted to have 4-5 orders of magnitude higher energy absorption and greater photostability than conventional photosensitizing dyes. ${ }^{14}$ Additional advantages of GNPs include ease of synthesis, straightforward conjugation to a variety of targeting molecules, ability to tune the optical properties to absorb at specific wavelengths, and utility in multimodal applications such as simultaneous imaging and treatment. ${ }^{14,15}$ Importantly, gold nanomaterials are also considered to exhibit relatively good biocompatibility, and therapies involving use of GNPs are currently undergoing testing in clinical trials. ${ }^{16}$

Previous studies have shown that use of GNPs with continuous wave or pulsed laser irradiation can significantly decrease the viability of several types of bacteria via photothermal cell lysis. ${ }^{9-12,17,18}$ Zharov et al ${ }^{10}$ proposed that the precision of microbial killing could be maximized and collateral host tissue damage minimized by combining nanomaterials functionalized with antibodies against specific bacterial cell wall components and nanosecond pulsed laser exposure. The antibody increases the specificity of nanoparticle binding, thereby targeting the thermally induced damage to the vicinity of the bacterial surface. Similarly, use of short laser pulses, compared to continuous wave irradiation, allows less time for heat diffusion to surrounding host tissue during exposure for more localized bacterial damage and reduced nonspecific damage to normal tissue; this may also allow integration of detection through photoacoustic or other mechanisms into the treatment platform. ${ }^{8,10,19}$ Using this approach, one research group reported a $\geq 95 \%$ killing of a methicillin-sensitive strain of $S$. aureus, in vitro, using laser pulses at $532 \mathrm{~nm}$ and a two-step nanoparticle targeting strategy involving binding of primary antibodies to the bacteria followed by treatment with secondary antibodies conjugated with gold nanospheres. ${ }^{10}$ The purpose of the current study was to test the antibacterial effect of this laser-induced photothermal technique against both methicillin-sensitive and methicillinresistant $S$. aureus using a simplified, one-step method for targeting of the GNPs to the bacteria.

\section{Materials and methods Growth of bacterial cultures}

Methicillin-sensitive Staphylococcus aureus (MSSA; ATCC $^{\circledR} 29213^{\mathrm{TM}}$ ) and methicillin-resistant S. aureus (MRSA; ATCC ${ }^{\circledR} 33591^{\mathrm{TM}}$ ) strains of $S$. aureus were obtained from the American Type Culture Collection (Manassas, VA, USA). Most of the experiments were conducted using only the MSSA strain to simplify sample handling and for experimental convenience. MSSA and MRSA cultures were grown aerobically in tryptic soy broth or nutrient broth (BD, Franklin Lakes, NJ, USA), respectively, on a shaking incubator at $250 \mathrm{rpm}$ and $37^{\circ} \mathrm{C}$ to an $\mathrm{OD}_{600}$ of $0.5-0.7$.

\section{Analysis of antibody binding to MSSA and MRSA}

Cultures were centrifuged at $5,000 \times g$ for 5 minutes at room temperature and the bacterial pellets were washed and resuspended in phosphate buffered saline (PBS; Gibco, Grand Island, NY, USA) with $0.1 \%$ bovine serum albumin (BSA; Sigma-Aldrich, St Louis, MO, USA). Biotinylated monoclonal antibodies specific to $S$. aureus peptidoglycan (1.3 mg/mL, Clone 702, Catalog Number BM3066B; Acris Antibodies, San Diego, CA, USA) were added to the samples at a 1:100 volumetric ratio. Control samples were prepared for both bacterial strains as described above, except no antibody was added. All samples were incubated for 1 hour at room temperature on an orbital mixer, then pelleted via centrifugation. Bacteria were washed twice using PBS with $0.1 \% \mathrm{BSA}$ and resuspended in the original volume in PBS containing $0.1 \%$ BSA, $1 \%$ goat serum (Jackson ImmunoResearch Laboratories, Inc., West Grove, PA, USA), and Alexa Fluor $^{\circledR}$ 488-AffiniPure Goat Anti-Mouse IgG $(1.5 \mathrm{mg} / \mathrm{mL}$, Catalog Number 115-545-146; Jackson ImmunoResearch Laboratories, Inc.) at a 1:400 dilution. After incubation for 1 hour at room temperature on an orbital mixer, samples were centrifuged, washed three times with PBS, and resuspended 
in PBS. Six aliquots $(100 \mu \mathrm{L})$ of each sample were placed into individual wells of a 96-well microtiter plate. A Synergy HT Microplate Reader (BioTek, Winooski, VT, USA) was used to read $\mathrm{OD}_{630}$ for the amount of bacteria and fluorescence at excitation/emission settings of $485 / 528 \mathrm{~nm}$ for the amount of bound antibody. The fluorescent signal was divided by the $\mathrm{OD}_{630}$ reading to standardize for the amount of bacteria in each sample. Standardized values for MSSA and MRSA from three independent experiments were compared using a Student's two-tailed $t$-test (STATISTICA ${ }^{\circledR}$, v10; StatSoft, Inc., Tulsa, OK, USA), with $P<0.05$ considered significant.

\section{Preparation of functionalized gold nanoparticles}

Sterile-filtered $40 \mathrm{~nm}$ gold nanospheres coated with streptavidin were obtained commercially (15 OD; BioAssay Works, Ijamsville, MD, USA). Aliquots containing approximately $6.5 \times 10^{10}$ nanoparticles were diluted with $1 \mathrm{~mL}$ of PBS containing $0.1 \%$ BSA and 10\% glycerol (Sigma-Aldrich; vehicle containing PBS, BSA, and glycerol hereafter referred to as PBG) and centrifuged at $7,400 \times g$ for 10 minutes at room temperature to remove the original vehicle. The supernatant was removed, the pellet was suspended in $0.5 \mathrm{~mL}$ of PBG, and biotinylated monoclonal antibodies specific to $S$. aureus peptidoglycan (Acris Antibodies) were added at a 1:100 volumetric ratio. Pilot testing using different volumetric ratios from $1: 50$ to $1: 50,000$ indicated $1: 100$ was the lowest antibody concentration required to provide maximal bacterial killing (data not shown). Nonfunctionalized gold nanospheres were prepared as described above for functionalized particles except antibody was not added. All samples were incubated for 1 hour at room temperature on an orbital mixer.

\section{Laser-induced photothermal killing of bacteria}

Bacterial cultures were centrifuged at $5,000 \times g$ for 5 minutes at room temperature, the supernatant was removed, and the bacterial pellets were suspended in half the original culture volume in PBG. Tubes containing either the functionalized or nonfunctionalized GNPs were removed from the orbital shaker and $0.5 \mathrm{~mL}$ of the bacterial suspension was added to each tube. For vehicle control samples, $0.5 \mathrm{~mL}$ of the bacterial suspension in PBG was added to a tube containing $0.5 \mathrm{~mL}$ of PBG without any nanoparticles. All tubes were then returned to the orbital mixer for 90 minutes of incubation at room temperature. Pilot experiments indicated antibody alone had no significant effect on MSSA colony forming ability (data not shown), and therefore this experimental group was not included in subsequent testing.

Laser exposures were performed using an Nd:YAG Q-switched laser (model CRF400; Big Sky/Quantel, Bozeman, MT, USA) with a wavelength of $532 \mathrm{~nm}$, an $8 \mathrm{~ns}$ pulse duration, and a pulse repetition rate of $1 \mathrm{~Hz}$. The optical system included a $250 \mathrm{~mm}$ focal length lens and a variable aperture arranged to provide a $2 \mathrm{~mm}$ diameter beam with a maximum energy density of approximately $5 \mathrm{~J} / \mathrm{cm}^{2} /$ pulse. ${ }^{10}$ The approximate size and alignment of the beam was confirmed using ZAP-IT ${ }^{\circledR}$ paper (Zap-It Corporation, Salisbury, NH, USA). Neutral density filters were placed in the beam path to reduce the pulse energy in the experiments conducted to test the effect of laser fluence on bacterial killing. A Nova II Laser Energy Meter with a Pyroelectric Energy Sensor (model PE25BF-DIF-C) and StarLab 2.0 software (all from Newport, Irvine, CA, USA) were used to measure and record the pulse energies for each experiment. Laser dosimetry was performed immediately pre- and postexposure by recording the energy of ten successive pulses. These pre- and postexposure values were combined to calculate the mean exposure energy, which was used to calculate laser fluence (energy per unit area) assuming a $2 \mathrm{~mm}$ beam diameter.

Triplicate or quadruplicate $75 \mu \mathrm{L}$ aliquots from each bacterial sample were exposed in quartz cuvettes with a $2 \mathrm{~mm}$ wide window and $10 \mathrm{~mm}$ light path (Precision Cells, Farmingdale, NY, USA). During exposures, cuvettes were placed in a holder with black side walls to minimize light scatter while allowing the laser beam to pass through the front and back windows. Vehicle controls and bacteria treated with GNPs alone were transferred to cuvettes and subjected to sham exposure for the approximate duration of laser treatment without laser activation. After irradiation with 100 pulses or sham exposure, the aliquots were gently mixed by pipetting, transferred to fresh tubes, serially diluted in PBS, and plated in triplicate on tryptic soy agar. Bacterial colonies were counted manually after overnight incubation at $37^{\circ} \mathrm{C}$. Data in graphs are expressed as percent survival compared to the vehicle controls and represent the mean and standard deviation (SD) of three to six independent experiments. Statistical analysis of results was conducted using repeated measures analysis of variance (ANOVA) tests followed by post hoc Tukey or Dunnett tests (STATISTICA ${ }^{\circledR}$; StatSoft, Inc.), with $P<0.05$ considered significant. ${ }^{20}$ Linear regression was performed using SigmaPlot ${ }^{\mathrm{TM}}$ (v10.0; Systat Software, Inc., San Jose, CA, USA). 


\section{Scanning electron microscopy}

Selected samples of MSSA were collected on $0.22 \mu \mathrm{M}$ filters immediately after sham or laser exposure. The bacteria on the filters were washed once in PBS and twice in double-distilled $\mathrm{H}_{2} \mathrm{O}$, fixed in $2.5 \%$ glutaraldehyde in PBS, and then dehydrated through a series of increasing alcohol concentrations. After mounting the filters on specimen stubs, samples were coated using a Hummer 6.2 Sputter Coater (Anatech USA, Union City, CA, USA) with a gold-palladium (50\%-50\%) target and imaged using a Sigma-VP40 field emission scanning electron microscope (Carl Zeiss Inc., Jena, Germany) in high vacuum mode.

\section{Results and discussion}

\section{Targeted photothermal destruction of MSSA}

Figure 1 shows the results of testing the antibacterial effect of nonfunctionalized or antibody-functionalized GNPs either alone or in combination with $532 \mathrm{~nm}$ pulsed laser irradiation against MSSA. Exposure of bacteria to nonfunctionalized or functionalized GNPs without laser treatment resulted in $88 \% \pm 8 \%$ and $79 \% \pm 5 \%$ (mean $\pm \mathrm{SD}, \mathrm{n}=4$ ) survival, respectively, relative to the vehicle control group. When laser irradiation was combined with nonfunctionalized GNPs, viability remained at $75 \% \pm 7 \%$ of the controls and was not significantly different from survival observed in the two experimental groups treated with nanoparticles alone. In contrast, use of GNPs coated with anti-S. aureus antibodies with pulsed laser

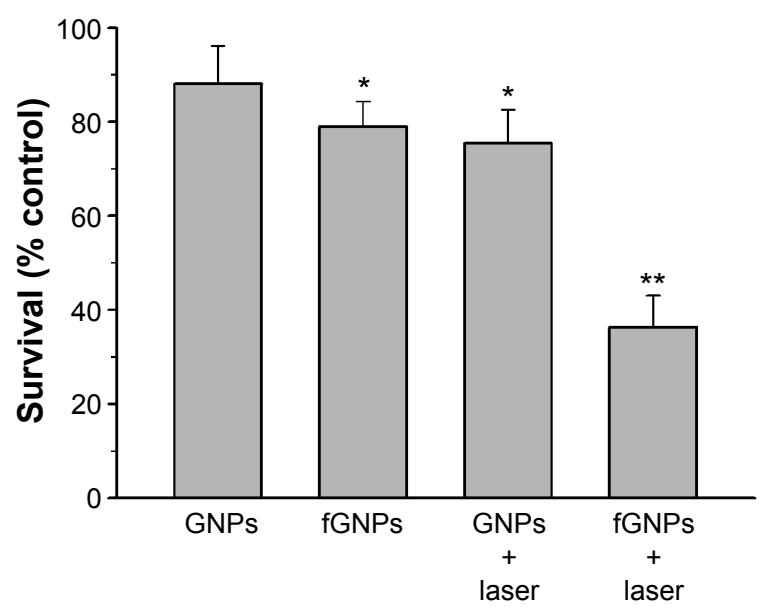

Figure I Targeted killing of MSSA using antibody-functionalized GNPs combined with pulsed laser irradiation at $532 \mathrm{~nm}$.

Notes: Laser irradiation consisted of 100 pulses at $5 \mathrm{~J} / \mathrm{cm}^{2}$. Bacterial survival was determined by colony forming unit assays. Survival in the vehicle control group, which did not receive nanoparticles or laser treatment, was set to $100 \%$. Values represent the mean + standard deviation of four independent experiments. $* P \leq 0.005$ compared to the control group and $* * P=0.0002$ compared to controls and the other three treatment groups as determined by repeated measures ANOVA followed by post hoc Tukey test. Abbreviations: ANOVA, analysis of variance; MSSA, methicillin-sensitive Staphylococcus aureus; GNPs, gold nanoparticles; fGNPs, functionalized gold nanoparticles. exposure decreased viability to $36 \% \pm 7 \%$ of controls, and this was significantly less than survival in the controls and other three treatment groups $(P=0.0002)$. Though the combination of nonfunctionalized GNPs and laser exposure caused a slight but significant reduction in viability compared to the control group, the results illustrate the positive influence of the targeting antibody on bacterial killing and are in agreement with prior reports showing use of a targeting moiety was necessary to induce pulsed laser-induced photothermal killing in bacteria and mammalian cells..$^{10,15}$

Selected MSSA samples were collected on filters for scanning electron microscopy (SEM) imaging to visually confirm attachment of antibody-functionalized GNPs to the bacteria and determine the presence of morphological changes indicative of laser-induced photothermal damage to the cells. Backscattered electron images of MSSA incubated with vehicle alone as the control, nonfunctionalized GNPS, or antibody-functionalized GNPs are shown in Figure 2A. In MSSA samples treated with nonfunctionalized GNPs, almost all of the visible particles, which show up as bright white dots, appear on the collection filter rather than in close contact with the bacteria. In contrast, a much greater number of GNPs appear attached to the bacteria when functionalized with the anti-S. aureus antibody. These findings are in line with the results reported in Figure 1 and further support the hypothesis of a highly localized photothermal effect generated by the pulsed laser around the nanoparticles, necessitating close contact between the GNPs and bacteria for optimal killing.

Secondary electron images of MSSA incubated with functionalized GNPs followed by sham or pulsed laser exposure are shown in Figure 2B. Sham exposed samples appear as typical clumps of cocci with GNPs attached individually or as clusters, whereas laser exposed samples indicate the presence of damaged bacteria that appear as shrunken or flattened cells. Mechanisms of pulsed laser-induced photothermal damage to bacteria may involve generation of acoustic and shock waves, bubble formation, thermal injury, and melting or fragmentation of the nanoparticles..$^{10,18,19}$ Not all of the bacteria showed overt signs of damage after laser exposure, including some that had nanoparticles attached to the surface. It is possible that the typical growth pattern of S. aureus as clumps may shield some of the bacteria from the incident laser energy or binding of a sufficient number of GNPs to induce complete killing.

\section{Effect of laser fluence on photothermal destruction of MSSA}

The antibacterial effect of targeted GNPs and pulsed laser irradiation was tested at varying laser fluences from 0 to $5 \mathrm{~J} / \mathrm{cm}^{2}$ 
A

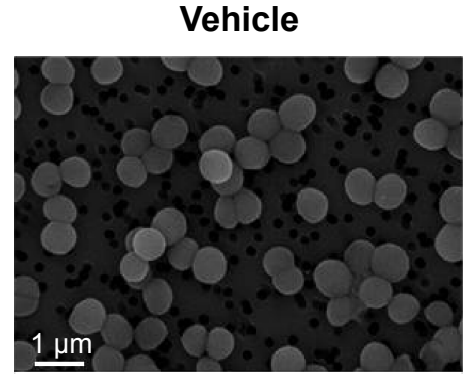

GNPs

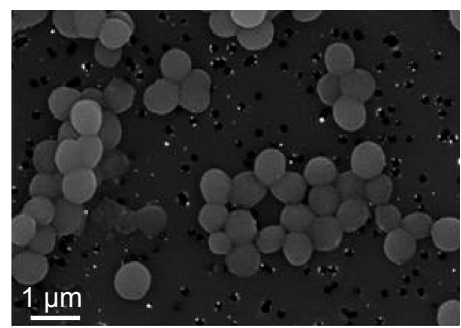

fGNPs

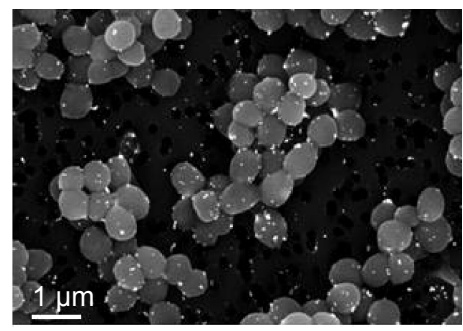

B

Sham exposure
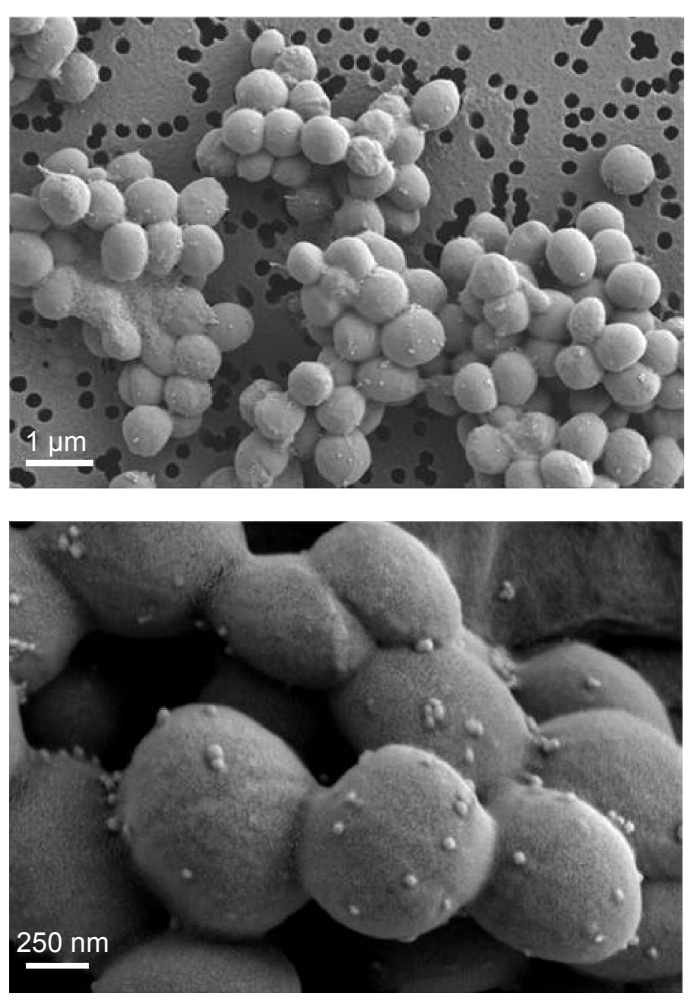

Laser exposure
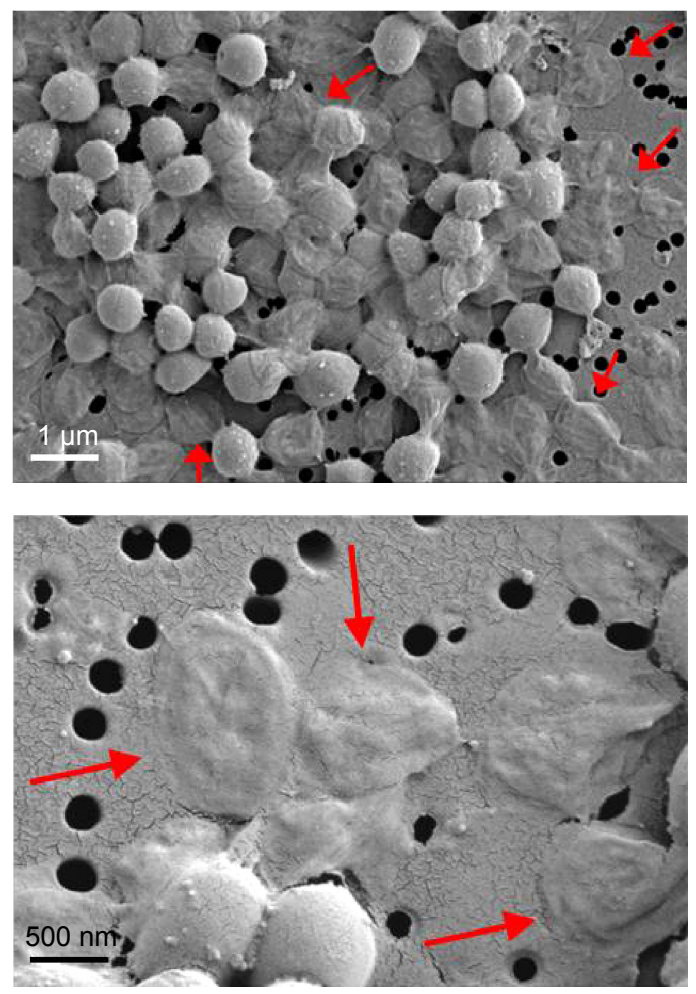

Figure 2 SEM images of MSSA.

Notes: (A) Backscattered electron images of MSSA treated with PBG vehicle, nonfunctionalized GNPs, or fGNPs. All images are at $25,000 \times$ magnification. Scale bars $=1$ mm. (B) Secondary electron images showing MSSA treated with fGNPs then sham exposed (left column) or exposed to pulsed laser irradiation at $532 \mathrm{~nm}$ (right column). Red

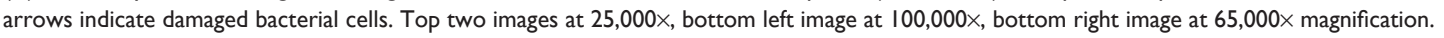

Abbreviations: SEM, scanning electron microscopy; MSSA, methicillin-sensitive Staphylococcus aureus; PBG, phosphate buffered saline with bovine serum albumin and glycerol; GNPs, gold nanoparticles; fGNPs, functionalized gold nanoparticles.

to characterize the dose-response relationship (Figure 3). Survival in the $0 \mathrm{~J} / \mathrm{cm}^{2}$ (sham exposed) group was $86 \% \pm 20 \%$ (mean $\pm \mathrm{SD}, \mathrm{n}=6$ ) compared to the vehicle controls, and increasing the laser dose from 1 to $5 \mathrm{~J} / \mathrm{cm}^{2}$ resulted in decreasing levels of viability from $75 \% \pm 11 \%$ to $31 \% \pm 8 \%$. Bacteria exposed to 2,3 , or $5 \mathrm{~J} / \mathrm{cm}^{2}$ had significantly lower survival rates compared to the $0 \mathrm{~J} / \mathrm{cm}^{2}$ group, whereas viability in the bacteria exposed to $1 \mathrm{~J} / \mathrm{cm}^{2}$ did not differ significantly from the sham exposed group. Regression analysis of the data revealed a linear relationship between photothermal killing and laser fluence with an $r^{2}$ value of 0.97 .

Overall, the data show a similar pattern of decreasing viability as that previously published for MSSA exposed to targeted GNPs and nanosecond pulsed laser irradiation at $532 \mathrm{~nm}$ from 0 to $5 \mathrm{~J} / \mathrm{cm}^{2} .{ }^{10}$ The prior report showed $\leq 10 \%$ bacterial survival for laser fluences at or above $3 \mathrm{~J} / \mathrm{cm}^{2}$, which is somewhat lower than that achieved here. This disparity may be due to use of different strains of $S$. aureus in the two investigations or use of a one-step approach 


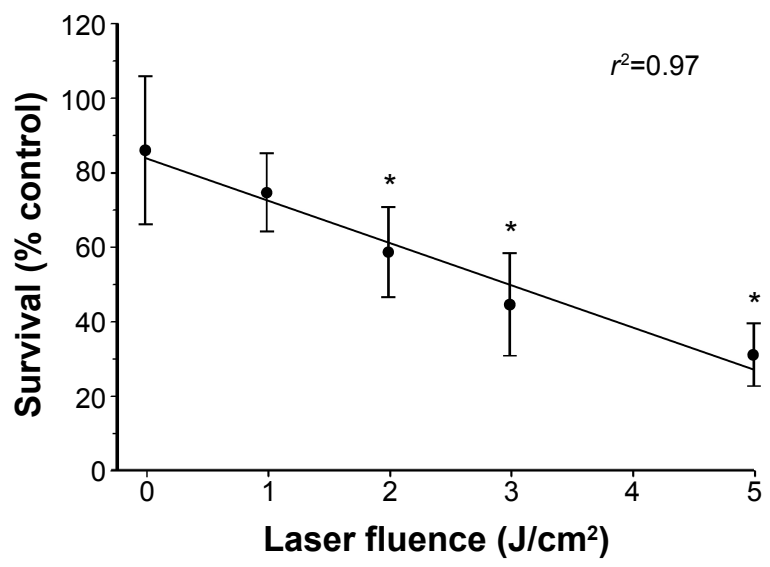

Figure 3 Linear relationship between laser-induced photothermal killing and laser fluence in MSSA.

Notes: MSSA samples were incubated with $40 \mathrm{~nm}$ gold nanospheres coated with anti-S. aureus antibodies then exposed to 100 laser pulses at varying laser fluences. Bacterial survival was determined by colony forming unit assays. The vehicle control group, which did not receive nanoparticles or laser treatment, was set to $100 \%$ survival. Values are expressed as mean \pm standard deviation of six independent experiments, and the fit line and associated $r^{2}$ value were derived from linear regression analysis. $* P \leq 0.00003$ compared to the $0 \mathrm{~J} / \mathrm{cm}^{2}$ group as determined by repeated measures ANOVA followed by post hoc Dunnett test.

Abbreviation: ANOVA, analysis of variance; MSSA, methicillin-sensitive Staphylococcus aureus.

for targeting the GNPs to the bacteria in the present study instead of the two-step method utilized previously. In addition, antibodies specific to peptidoglycan were employed in the current investigation, rather than anti-protein A antibodies as used in the earlier study, because growth phase- and S. aureus strain-dependent variations in protein A expression may affect efficacy of antibody-targeted antimicrobial therapies. $^{21}$

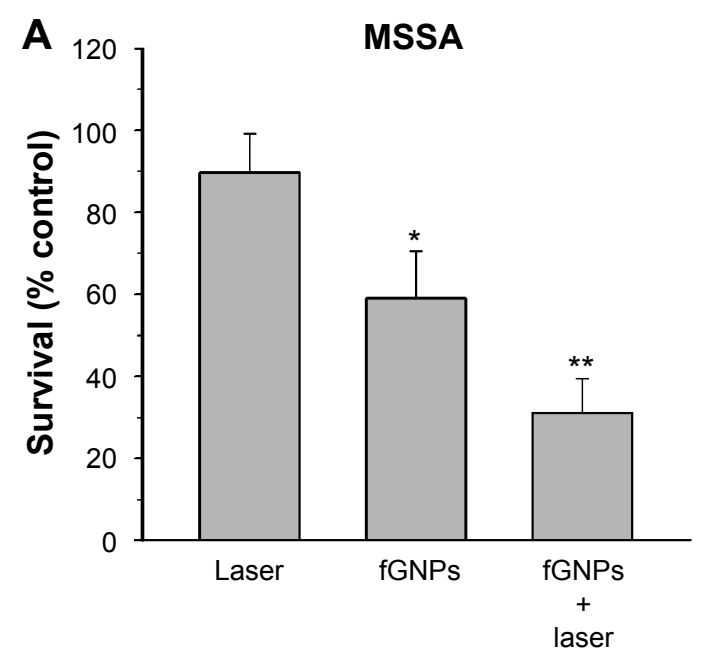

\section{Efficacy of photothermal killing against MSSA and MRSA}

The efficacy of the pulsed laser-induced photothermal treatment was tested against an MRSA strain in addition to the MSSA strain used in the above experiments. As shown in Figure 4, laser exposure alone did not induce a significant reduction in viability in either strain compared to the vehicle control group. Incubation with functionalized GNPs alone did not significantly affect viability of the MRSA strain (Figure 4B) but decreased survival to $59 \% \pm 11 \%$ (mean \pm $\mathrm{SD}, \mathrm{n}=3$ ) in the MSSA strain, though this was of borderline statistical significance with $P=0.04$. The combination of functionalized GNPs and pulsed laser exposure, however, caused a significant reduction in survival to $31 \% \pm 8 \%$ and $58 \% \pm 10 \%$, respectively, in the MSSA and MRSA strains. Though comparison of results from the two strains indicate a potential difference in sensitivity, the data from MSSA and MRSA were not compared statistically because testing for MSSA and MRSA was conducted in separate experimental runs.

Differential sensitivity of these two bacterial strains could be due to multiple factors including variations in the epitope to which the antibody binds and other structural characteristics affecting the inherent sensitivity of the cells to photothermal effects. Increased cell wall thickness, for example, has been associated with antibiotic resistance in $S$. aureus ${ }^{22,23}$ and could decrease susceptibility of MRSA to photoacoustic damage. In addition, variations in surface antigenicity of MSSA and MRSA ${ }^{24,25}$ could influence the affinity of the

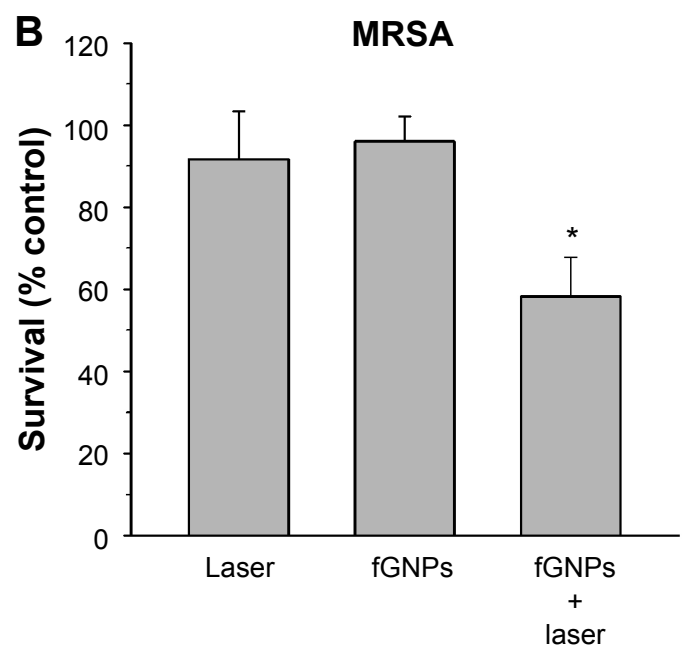

Figure 4 Antibacterial effect of targeted gold nanoparticles and pulsed laser irradiation at $532 \mathrm{~nm}$ against (A) MSSA and (B) MRSA.

Notes: Bacterial samples were incubated with $40 \mathrm{~nm}$ gold nanospheres coated with anti-S. aureus antibodies then exposed to 100 laser pulses at $5 \mathrm{~J} / \mathrm{cm}^{2}$. Bacterial survival was determined by colony forming unit assays. The vehicle control group, which did not receive gold nanoparticles or laser treatment, was set to $100 \%$ survival. Values are expressed as mean + standard deviation of three independent experiments. Statistical significance was determined using repeated measures ANOVA tests and post hoc Tukey tests. (A) $* P=0.04$ compared to the controls and $* * P \leq 0.005$ compared to the controls and laser alone group. (B) $* P \leq 0.01$ compared to the controls and the other two treatment groups. Abbreviations: ANOVA, analysis of variance; MSSA, methicillin-sensitive Staphylococcus aureus; MRSA, methicillin-resistant Staphylococcus aureus; fGNPs, functionalized gold nanoparticles. 
targeting antibody to the individual strains leading to differences in sensitivity. With this possibility in mind, binding of the biotinylated anti-S. aureus primary antibody to the MSSA and MRSA strains was tested using a fluorescently labeled anti-mouse secondary antibody in a microtiter plate assay. Values for fluorescence intensity standardized to $\mathrm{OD}_{630}$ were 249,366 $\pm 37,294$ and 276,922 $\pm 16,189$ (mean $\pm S D, n=3$ ) for MRSA and MSSA, respectively, and were not significantly different $(P=0.31)$. These results indicate that differences in the level of binding of the antibody to the bacteria do not account for the variation in killing between strains observed in this study. However, further testing using a larger panel of bacterial strains and antibodies against other surface antigens is required to determine if true variations exist in the efficacy of the laser-induced photothermal treatment against different strains of $S$. aureus.

\section{Conclusion}

In conclusion, our findings support the use of laser-activated, functionalized GNPs as an adjunct therapy for $S$. aureus infections. The treatment can be targeted to specific organisms, and the bactericidal effect is linearly related to the laser fluence. While not completely eliminating all viable bacteria, at least in the treatment's present form, this novel therapy has a potential application as an adjunct to conventional therapy for reducing the infectious load in a lesion, thus facilitating the complete eradication with the concomitant administration of a suitable antibiotic. Because the systemic elimination of nanoparticles is incompletely understood at present, ${ }^{26}$ this therapy is most suited for treatment of cutaneous infections, in which the subsequent removal of the particles following the treatment is not a serious concern. Finally, the efficacy of the nanoparticle therapy may likely be optimized in future studies by adjusting the size and shape of the nanoparticles and the laser parameters to regulate the nature of the induced shock waves, the extent of thermal diffusion from the metal particle, and laser-tissue interactions. It may also be possible to improve the therapy by modifying the particular bacterial antigen targeted by the functionalizing ligand on the nanoparticle.

\section{Acknowledgments}

This work was supported by the Naval Medical Research Unit San Antonio work unit number G1025. The authors gratefully acknowledge Dr Tao You of the US Army Dental Trauma and Research Detachment at the Institute of Surgical Research for assistance with SEM imaging. The views expressed in this article are those of the authors and do not necessarily reflect the official policy or position of the Department of the Navy, Department of Defense, nor the US Government. NJM, JBB, WRE, and MND are employees of the US Government. This work was prepared as part of their official duties. Title 17 USC $§ 105$ provides that 'Copyright protection under this title is not available for any work of the United States Government.' Title 17 USC $\$ 101$ defines a US Government work as a work prepared by a military service member or employee of the US Government as part of that person's official duties.

\section{Disclosure}

The authors report no conflicts of interest in this work.

\section{References}

1. Boucher HW, Corey GR. Epidemiology of methicillin-resistant Staphylococcus aureus. Clin Infect Dis. 2008;46(Suppl 5):S344-S349.

2. Stryjewski ME, Chambers HF. Skin and soft-tissue infections caused by community-acquired methicillin-resistant Staphylococcus aureus. Clin Infect Dis. 2008;46(Suppl 5):S368-S377.

3. Talan DA, Krishnadasan A, Gorwitz RJ, et al. Comparison of Staphylococcus aureus from skin and soft-tissue infections in US emergency department patients, 2004 and 2008. Clin Infect Dis. 2011;53(2):144-149.

4. Lee BY, Singh A, David MZ, et al. The economic burden of communityassociated methicillin-resistant Staphylococcus aureus (CA-MRSA). Clin Microbiol Infect. 2013;19(6):528-536.

5. Moran GJ, Abrahamian FM, Lovecchio F, Talan DA. Acute bacterial skin infections: developments since the 2005 Infectious Diseases Society of America (IDSA) guidelines. J Emerg Med. 2013;44(6):e397-e412.

6. Edelsberg J, Taneja C, Zervos M, et al. Trends in US hospital admissions for skin and soft tissue infections. Emerg Infect Dis. 2009;15(9):1516-1518.

7. Otter JA, French GL. Community-associated meticillin-resistant Staphylococcus aureus strains as a cause of healthcare-associated infection. J Hosp Infect. 2011;79(3):189-193.

8. Galanzha EI, Shashkov E, Sarimollaoglu M, et al. In vivo magnetic enrichment, photoacoustic diagnosis, and photothermal purging of infected blood using multifunctional gold and magnetic nanoparticles. PLoS One. 2012;7(9):e45557.

9. Kim JW, Shashkov EV, Galanzha EI, Kotagiri N, Zharov VP. Photothermal antimicrobial nanotherapy and nanodiagnostics with self-assembling carbon nanotube clusters. Lasers Surg Med. 2007;39(7):622-634.

10. Zharov VP, Mercer KE, Galitovskaya EN, Smeltzer MS. Photothermal nanotherapeutics and nanodiagnostics for selective killing of bacteria targeted with gold nanoparticles. Biophys J. 2006;90(2): 619-627.

11. Norman RS, Stone JW, Gole A, Murphy CJ, Sabo-Attwood TL. Targeted photothermal lysis of the pathogenic bacteria, Pseudomonas aeruginosa, with gold nanorods. Nano Lett. 2008;8(1):302-306.

12. Huang WC, Tsai PJ, Chen YC. Functional gold nanoparticles as photothermal agents for selective-killing of pathogenic bacteria. Nanomedicine (Lond). 2007;2(6):777-787.

13. Dai T, Tegos GP, Zhiyentayev T, Mylonakis E, Hamblin MR. Photodynamic therapy for methicillin-resistant Staphylococcus aureus infection in a mouse skin abrasion model. Lasers Surg Med. 2010; 42(1):38-44.

14. Jain PK, Lee KS, El-Sayed IH, El-Sayed MA. Calculated absorption and scattering properties of gold nanoparticles of different size, shape, and composition: applications in biological imaging and biomedicine. J Phys Chem B. 2006;110(14):7238-7248. 
15. Day ES, Bickford LR, Slater JH, Riggall NS, Drezek RA, West JL. Antibody-conjugated gold-gold sulfide nanoparticles as multifunctional agents for imaging and therapy of breast cancer. Int J Nanomedicine. 2010;5:445-454.

16. Cobley CM, Au L, Chen J, Xia Y. Targeting gold nanocages to cancer cells for photothermal destruction and drug delivery. Expert Opin Drug Deliv. 2010;7(5):577-587.

17. Kim CB, Yi DK, Kim PS, Lee W, Kim MJ. Rapid photothermal lysis of the pathogenic bacteria, Escherichia coli using synthesis of gold nanorods. J Nanosci Nanotechnol. 2009;9(5):2841-2845.

18. Wang S, Singh AK, Senapati D, Neely A, Yu H, Ray PC. Rapid colorimetric identification and targeted photothermal lysis of Salmonella bacteria by using bioconjugated oval-shaped gold nanoparticles. Chemistry. 2010;16(19):5600-5606.

19. Letfullin RR, Joenathan C, George TF, Zharov VP. Laser-induced explosion of gold nanoparticles: potential role for nanophotothermolysis of cancer. Nanomedicine (Lond). 2006;1(4):473-480.

20. Lew M. Good statistical practice in pharmacology. Problem 2. $\mathrm{Br}$ J Pharmacol. 2007;152(3):299-303.
21. Embleton ML, Nair SP, Cookson BD, Wilson M. Antibody-directed photodynamic therapy of methicillin resistant Staphylococcus aureus. Microb Drug Resist. 2004;10(2):92-97.

22. Kawai M, Yamagishi J. Mechanisms of action of acriflavine: electron microscopic study of cell wall changes induced in Staphylococcus aureus by acriflavine. Microbiol Immunol. 2009;53(9):481-486.

23. Morikawa K, Maruyama A, Inose Y, Higashide M, Hayashi H, Ohta T. Overexpression of sigma factor, sigma(B), urges Staphylococcus aureus to thicken the cell wall and to resist beta-lactams. Biochem Biophys Res Commun. 2001;288(2):385-389.

24. Shakeri F, Shojai A, Golalipour M, Rahimi Alang S, Vaez H, Ghaemi EA. Spa Diversity among MRSA and MSSA Strains of Staphylococcus aureus in North of Iran. Int J Microbiol. 2010;2010: pii: 351397.

25. Rudkin JK, Edwards AM, Bowden MG, et al. Methicillin resistance reduces the virulence of healthcare-associated methicillin-resistant Staphylococcus aureus by interfering with the agr quorum sensing system. J Infect Dis. 2012;205(5):798-806.

26. Almeida JP, Chen AL, Foster A, Drezek R. In vivo biodistribution of nanoparticles. Nanomedicine (Lond). 2011;6(5):815-835.
International Journal of Nanomedicine

\section{Publish your work in this journal}

The International Journal of Nanomedicine is an international, peerreviewed journal focusing on the application of nanotechnology in diagnostics, therapeutics, and drug delivery systems throughout the biomedical field. This journal is indexed on PubMed Central, MedLine, CAS, SciSearch ${ }^{\circledR}$, Current Contents ${ }^{\circledR} /$ Clinical Medicine,

\section{Dovepress}

Journal Citation Reports/Science Edition, EMBase, Scopus and the Elsevier Bibliographic databases. The manuscript management system is completely online and includes a very quick and fair peer-review system, which is all easy to use. Visit http://www.dovepress.com/ testimonials.php to read real quotes from published authors. 